\title{
Medical Students' Syndrome: A Cross Sectional Study from South East of Iran
}

\author{
Mahin Eslami, ${ }^{1}$ Habibeh Ahmadipour,, ${ }^{2,}$ and Faezeh Bagheri ${ }^{3}$ \\ ${ }^{1}$ Neurology Research Center, School of Medicine, Kerman University of Medical Sciences, Kerman, Iran \\ ${ }^{2}$ Social Determinants of Health Research Center, Institute for Futures Studies in Health, Kerman University of Medical Sciences, Kerman, Iran \\ ${ }^{3}$ School of Medicine, Kerman University of Medical Sciences, Kerman, Iran \\ Corresponding author: Habibeh Ahmadipour, Associate Professor of Community Medicine, Social Determinants of Health Research Center, Institute for Futures Studies in \\ Health, Kerman University of Medical Sciences, Kerman, Iran. Tel: +98-3433257313, Fax: +98-3433257671, E-mail: ahmadipour@kmu.ac.ir
}

Received 2017 December 15; Revised 2018 June 22; Accepted 2018 June 22.

Keywords: Hypochondriasis, Medical Student, Mental Health, Iran

\section{Dear Editor,}

Medical Students Syndrome (MSS) is defined as medical students experience health related anxiety while studying a specific disease due to the fact that they falsely related their symptoms to the disease being studied. This phenomenon is a kind of acute hypochondriasis and more common among medical students compared to other college students (1). Some of previous literatures revealed 70\% to $80 \%$ of medical students experienced MSS (2), while others found that MSS is not more likely in medical students compared to other students (3).

Studying medicine is a stressful process with probable negative physical and mental health consequences and likely poor academic performance among medical students (4). This stress on one hand and obtaining medical knowledge on the other hand causes medical students to relate previously neglected symptoms to the newly obtained knowledge (5). Limited studies in the Eastern Mediterranean region revealed that the prevalence of the disorder varies from $11.9 \%$ to $16 \%(6,7)$. The present study aimed to investigate the frequency and related factors of medical students' syndrome among medical students in Kerman University of medical sciences (KMU), Kerman province, Iran.

A cross sectional study was conducted in the medical school of KMU. All medical students who were studying in the clerkship and internship during 2016 were selected by the Census method.

The study was approved by the research review board (Ethic code: IR-KMUREC. 94.71). The inclusion criteria included being in the internship or clerkship for at least 6 month at the time of the study and informed consent to participate. The exclusion criteria included the questionnaires with more than $10 \%$ unanswered questions.

Data was collected using a self - administered two - section questionnaire including demographic data and the Persian versions of 14 - item of the Whiteley Index (WI). WI has been developed by Pilowsky and used in different populations. Psychometric properties of the Persian version of the instruments were confirmed in the previous study. A five - point Likert scale was used for responses on WI (1: not at all and 5: extremely). Therefore, a total score range from 14 to 70 was obtained; the higher score indicates greater severity of the health anxiety (8). The $90^{\text {th }}$ percentile of the questionnaire (63) was considered as a cut off point (9).

The questionnaires were distributed and taken back before or after weekly formal classes. The time required to complete the questionnaire was a maximum of $10 \mathrm{~min}$ utes. The questionnaires were completed anonymously and voluntarily. Data was analyzed by the SPSS version 19 using logistic regression and Chi-Square.

A total of 310 medical students participated in the study (response rate: $76.1 \%$ ). The mean of the participants' age was $24.11 \pm 1.50$ years. Of the respondents, $186(60 \%)$ were in the clerkship stage and 233 (75.2\%) were single.

The mean of the score on Whiteley index was $48.50 \pm$ 8.73. Accordingly, 34 (11.0\%) medical students had probable MSS. The frequency of probable MSS was significantly higher in the interns (15.3\%) than clerkship participant $(8.1 \%)(\mathrm{P}=0.04)$. Table 1 shows the frequency of probable MSS according to demographic data.

In logistic regression, no significant predictor was found for the presence of probable MSS.

The present study revealed that $11 \%$ of our medical students in clinical stages (clerkship and internship) had 


\begin{tabular}{|c|c|c|c|}
\hline Variables & With Probable Hypochondriasis & Without Probable Hypochondriasis & Pvalue \\
\hline Educational level & & & 0.04 \\
\hline Internship & $19(15.3)$ & $105(84.7)$ & \\
\hline Clerkship & $15(8.1)$ & $171(91.9)$ & \\
\hline Marital status & & & 0.54 \\
\hline Married & $7(9.1)$ & $70(90.9)$ & \\
\hline Parents' job & & & 0.58 \\
\hline Related to the medicine & $8(12.9)$ & $54(87.1)$ & \\
\hline Non-related to the medicine & $26(10.5)$ & $222(89.5)$ & \\
\hline Monthly household income (USD) & & & 0.27 \\
\hline Equal or more than 250 & $31(11.6)$ & $228(88.4)$ & \\
\hline
\end{tabular}

probable MSS. This finding is compatible with recent studies, which reported lower prevalence of the disorder while more previous studies reported a prevalence of over $70 \%$ (1$3)$. The discrepancy in the reported prevalence of this disorder may be due to using different diagnostic tools and differences in ethno - cultural construct.

In the present study, MSS was significantly higher in interns than clerkship students. Ferguson et al., found that whatever student's medical knowledge increase, the severity of disorder would increases, while Hardy and Calhoun showed contrary to this (10).

Due to the impact of the disorder on student academic performance, it is imperative that the educational system consider interventions to increase students' awareness regarding MSS. Therefore, it is essential that students' mental health status be evaluated periodically, which provides timely diagnosis and appropriate interventions as well as prevents negative consequences on the students' academic performance.

Our study was a cross - sectional study. Another limitation was that the data collection was self - reported by the trainees, which does not necessarily yield precise evidence. However, it was probably the first study that assessed MSS in our country. We also need to raise the awareness of the students in regards to the syndrome.

\section{Acknowledgments}

The authors are grateful to all medical students whom their cooperation, made this study possible.

\section{Footnotes}

Conflict of Interest: The authors had no conflict of interest.
Funding/Support: Kerman University of Medical Sciences supported this study.

\section{References}

1. Mahendran S, Jothipriya A. A comparative study on 2 nd year syndrome among dental medical and nursing students. Int J Curr Adv Res. 2017;6(3):2954-7. doi:10.24327/ijcar.2017.2957.0152.

2. Moss-Morris R, Petrie KJ. Redefining medical students' disease to reduce morbidity. Med Educ. 2001;35(8):724-8. [PubMed: 11489098].

3. Collier R. Imagined illnesses can cause real problems for medical students. Can Med Assoc J. 2008;178(7):820. doi:10.1503/cmaj.080316.

4. Waterman LZ, Weinman JA. Medical student syndrome: fact or fiction? A cross-sectional study. JRSM Open. 2014;5(2):1-9. doi: $10.1177 / 2042533313512480$.

5. Gupta S, Choudhury S, Das M, Mondol A, Pradhan R. Factors causing stress among students of a medical college in Kolkata, India. Educ Health (Abingdon). 2015;28(1):92-5. doi: 10.4103/1357-6283.161924. [PubMed: 26261123].

6. Talaei A. P02-290 Hypochondriasis in medical sciences students of Mashhad, Iran. Eur Psychiatr. 2009;24. S980. doi: 10.1016/s09249338(09)71213-1.

7. Zahid MF, Haque A, Aslam M, Aleem NA, Hussain S, Fahad H, et al Health-Related Anxiety and Hypochondriacal Concerns in Medical Students: A Cross-Sectional Study From Pakistan. Teach Learn Med. 2016;28(3):252-9. doi: 10.1080/10401334.2016.1155459.

8. Eslami M, Ahmadipour H, Bagheri F. Psychometric properties of the Persian version of Whiteley Index. Russ Open Med J. 2017;6(3). e0307. doi:10.15275/rusomj.2017.0307.

9. Berge LI, Skogen JC, Sulo G, Igland J, Wilhelmsen I, Vollset SE, et al. Health anxiety and risk of ischaemic heart disease: a prospective cohort study linking the Hordaland Health Study (HUSK) with the Cardiovascular Diseases in Norway (CVDNOR) project. BM] Open. 2016;6(11). e012914. doi: 10.1136/bmjopen-2016-012914. [PubMed: 27810977]. [PubMed Central: PMC5129078].

10. Candel I, Merckelbach H. Fantasy proneness and thought suppression as predictors of the Medical Student Syndrome. Pers Indiv Differ. 2003;35(3):519-24. doi:10.1016/s0191-8869(02)00214-3. 